treated with phlebotomy. We aimed to test HFE p.C282Y homozygote associations with prevalent and incident morbidity in the large UK Biobank sample of European descent. We also examined how iron supplement use may affect associations between p.C282Y homozygosity and morbidity.

Methods We studied 451,243 participants of European descent (aged 40 to 70 years) from the UK Biobank. Data were available on prevalent and incident adverse health outcomes from baseline questionnaires and from up to 9.4 years hospital inpatient follow-up (mean 7 years). Participants also reported baseline dietary supplement use. We tested associations between p.C282Y homozygosity, prevalent and incident outcomes, and iron supplement use, using logistic regression and Cox proportional hazard regression, adjusted for age, sex, genotyping array type and genetic principal components.

Results 2,890 participants were p.C282Y homozygotes $(0.6 \%$, or $1 / 156)$, of whom $7.3 \%(210 / 2890)$ had haemochromatosis diagnosed at baseline, increasing to $15.1 \%$ (437/2890) by the end of follow-up. P.C282Y homozygotes had substantial excess prevalent and incident morbidity including haemochromatosis, liver disease, arthritis and diabetes compared to those with no mutations (combined measure of excess incident morbidity; men, HR: 3.37, 95\% CI: 2.87-3.97; women, HR: 2.99,95\% CI: 2.51-3.55). A sub-analysis of 200,975 older participants (aged 60-70 years) showed that both male and female p. C282Y homozygotes also had an increased likelihood of Fried frailty and chronic pain.

In p.C282Y homozygotes undiagnosed with haemochromatosis, the intake of iron supplements or multivitamins increased the likelihood of frailty (OR: 2.15, 95\% CI: $1.22-$ 3.77 ) and incident osteoarthritis (HR: 1.86, 95\% CI: 1.023.41)

Conclusion In a large community volunteer sample, HFE p. $\mathrm{C} 282 \mathrm{Y}$ homozygosity was associated with substantial excess morbidity, frailty and chronic pain in both men and women. In p.C282Y homozygotes undiagnosed with haemochromatosis, taking iron supplements or multivitamins was an additional risk factor for developing morbidity, including frailty and osteoarthritis. Since the p.C282Y associated iron overload can be prevented and treated, these findings suggest there is a need for expanded case finding and screening for hereditary haemochromatosis. It also suggests that warnings and controls on iron containing supplements may be needed.

\section{OP38 PREDICTING THE RISK OF CHILDHOOD OVERWEIGHT AND OBESITY AT 4-5 YEARS USING PREGNANCY AND EARLY LIFE HEALTHCARE DATA}

${ }^{1} \mathrm{~N}$ Ziauddeen*, 'S Wilding, 'PJ Roderick, ${ }^{2,3} \mathrm{NS}$ Macklon, 1,4NA Alwan. ${ }^{1}$ School of Primary Care and Population Sciences, Faculty of Medicine, University of Southampton, Southampton, UK; ${ }^{2}$ Department of Obstetrics and Gynaecology, University of Copenhagen, Roskilde, Denmark; ${ }^{3}$ London Women's Clinic, London, UK; ${ }^{4}$ NIHR Southampton Biomedical Research Centre, University of Southampton and University Hospital Southampton NHS Foundatio, Southampton, UK

\subsection{6/jech-2019-SSMabstracts.38}

Background In England, 9.5\% of children aged 4-5 years and $20.1 \%$ aged $10-11$ years are obese, with the prevalence in the most deprived areas being more than twice as that in the least deprived. There is evidence illustrating the developmental origins of obesity, but it focuses on individual risk factors and comes mostly from research birth cohorts which are not necessarily representative of the wider population. There is no system-based early identification of childhood obesity risk at pregnancy stage and onwards. The aim was to develop and validate a risk identification system for childhood obesity using existing routinely collected maternal and early-life populationlevel healthcare data in Hampshire.

Methods Studying Lifecourse Obesity PrEdictors (SLOPE) study is an anonymised population-based linked cohort of maternal antenatal and delivery records for all births taking place at University Hospital Southampton 2003-2018, and child health records including information on postnatal growth, type of feeding and childhood body mass index (BMI) up to 14 years. Childhood age- and sex- adjusted BMI at 4-5 years was used to define the outcome of overweight and obesity in the models. Logistic regression models together with multivariable fractional polynomials were used to select model predictors and to identify transformations of continuous predictors that best predict the outcome. Predictive accuracy was evaluated by assessing model discrimination and calibration.

Results Childhood BMI was available for approximately 30000 children aged 4-5 years (9\% obese). Models were developed in stages, incorporating data collected at first antenatal booking appointment, birth and early life predictors. The area under the curve (AUC) was lowest (0.64) for the model only incorporating maternal predictors from the booking appointment and highest for the model incorporating all factors up to weight at 2 years for predicting outcome at 4-5 years (0.82 for overweight and obesity and 0.89 for obesity excluding overweight). Maternal predictors included BMI, smoking status at first antenatal appointment, age and ethnicity. Early life predictors included birthweight, gender, breastfeeding and weight at 1 or 2 years of age. Although AUC was lower for the booking models, maternal predictors remained consistent across the models, thus high-risk groups could be identified at an early stage with more precise estimation as the child grows.

Conclusion This prediction modelling can be used to identify and quantify clustering of risk for childhood obesity as early as the first trimester of pregnancy, and can strengthen the long-term preventive element of antenatal and early years care.

\section{OP39 DEVELOPMENT OF A SHORT FOOD FREQUENCY QUESTIONNAIRE TO ASSESS DIET QUALITY IN POPULATION STUDIES}

${ }^{1}$ SR Crozier*, ${ }^{2,3}$ SM Robinson, ${ }^{1,4}$ S Shaw, ${ }^{1,4} \mathrm{HM}$ Inskip, ${ }^{1,4}$ J Baird, ${ }^{1,4} \mathrm{C}$ Cooper, ${ }^{1,4} \mathrm{C}$ Vogel. ${ }^{1} M R C$ Lifecourse Epidemiology Unit, University of Southampton, Southampton, UK; ${ }^{2} A G E$ Research Group, Newcastle University, Newcastle upon Tyne, UK; ${ }^{3}$ NIHR Newcastle Biomedical Research Centre, Newcastle upon Tyne Hospitals NHS Foundation Trust and Newcastle University, Newcastle upon Tyne, UK; ${ }^{4}$ NIHR Southampton Biomedical Research Centre, University of Southampton and University Hospital Southampton NHS Trust, Southampton, UK

\subsection{6/jech-2019-SSMabstracts.39}

Background Food frequency questionnaires (FFQs) are a popular tool in nutritional epidemiology, enabling estimates of habitual diet in large populations, but are time-consuming to complete. There is an increasing need for a short, accurate dietary tool that characterises healthy dietary patterns for use in observational and interventional research.

Methods The National Diet and Nutrition Survey (NDNS) is a general population national survey. Randomly-selected 
participants from across the UK were asked to keep a diary of everything they ate and drank over four days. Diaries were coded and intake frequencies of foods derived using groups of nutritionally similar foods. For the $2 \%$ of participants who had three-day diaries, the frequencies were multiplied by $4 / 3$. Principal component analysis (PCA) was performed on frequencies of consumption. Reduced-item prudent diet scores were calculated by multiplying coefficients for the ten most characteristic foods by each individual's standardized reported frequency of consumption. Prudent diet scores calculated for both the full and 10-item prudent diet scores were standardised. Willing participants provided a blood sample; vitamin C status was measured on the BMG Labtech FLUOstar OPTIMA plate reader and beta carotene status by high performance liquid chromatography.

Results 6090 adults (aged 20 to 96 years) provided dietary data in the first eight years of the NDNS (2008-2016). PCA of intake frequencies of 126 food groups revealed a prudent diet pattern characterised by high intakes of wholemeal bread, vegetables, fruit, water, oily fish, yoghurt and high-fibre breakfast cereals, and low intakes of white bread, sugar, sugarsweetened soft drinks and chips. Pattern coefficients were very similar when analyses were conducted separately in males and females, and in those aged less than 65 and $65+$ years. The 10 -item prudent diet score was calculated based on foods with the five highest and five lowest coefficients. Spearman's correlation between the full and 10-item prudent diet scores was 0.84 . The mean difference between the full and 10-item prudent diet scores was 0.00 SDs with Bland-Altman limits of agreement -1.89 to $1.89 \mathrm{SD}$. Notable correlations were seen between the full prudent diet score and vitamin $C\left(r_{s}=0.43\right)$ and beta-carotene status $\left(r_{s}=0.45\right)$; these were only slightly attenuated for the 10 -item prudent diet score $\left(r_{s}=0.40\right.$, $\mathrm{r}_{\mathrm{s}}=0.38$ respectively).

Conclusion A 10-item prudent diet score based on foods that characterise the prudent dietary pattern describes a healthy diet. A 10-item FFQ would have clear advantages for time and resources, and may provide an appropriate tool to describe UK diets while reducing participant burden.

\section{OP108 A PROSPECTIVE COHORT STUDY ON NUT AND PEANUT BUTTER INTAKE AND THE RISK OF ENDOMETRIAL AND OVARIAN CANCER}

'L Nieuwenhuis*, 1,2PA van den Brandt. 'Epidemiology, Care and Public Health Research Institute (CAPHRI), Maastricht University, Maastricht, Netherlands; ${ }^{2}$ Epidemiology, GROW School for Oncology and Developmental Biology, Maastricht University, Maastricht, Netherlands

\subsection{6/jech-2019-SSMabstracts.40}

Background Nut intake has been associated with reduced cancer-related mortality and cancer risk. However, very few studies investigated the association between nut consumption and the risk of endometrial and ovarian cancer, with inconclusive results. We prospectively examined the relation between total nut, tree nut, peanut, and peanut butter intake and the risk of endometrial and ovarian cancer in the prospective Netherlands Cohort Study (NLCS).

Methods In 1986, 62,573 women aged 55-69 years were included in the NLCS. At baseline, all participants filled in a questionnaire and a subcohort of 2,589 women was randomly selected. After 20.3 years of follow-up, 389 endometrial and 347 ovarian cancer cases with complete data were included in the analysis. Hazard ratios (HRs) were calculated in multivariable-adjusted Cox regression analyses, using a case-cohort approach.

Results Compared to nonconsumers, the HRs (95\% confidence intervals) for women consuming $10+\mathrm{g}$ total nuts/day were $1.23(0.82-1.87)$ for endometrial cancer and $0.84(0.57-1.24)$ for ovarian cancer. For tree nut, peanut, and peanut butter intake, also no significant relations with endometrial or ovarian cancer were observed. In the endometrial cancer analyses, significant interactions of total nut intake with body mass index and cigarette smoking status were found.

Conclusion The results of this study suggest that intake of total nuts, tree nuts, peanuts, and peanut butter is not related to the risk of endometrial or ovarian cancer. The observed interactions in the endometrial cancer analyses, particularly by cigarette smoking status, require confirmation in other studies.

\section{Health Inequalities 1}

\section{OP40 THE ASSOCIATION BETWEEN CHILDHOOD SOCIO- ECONOMIC POSITION AND ADVERSE CHILDHOOD EXPERIENCES (ACES): A SYSTEMATIC REVIEW}

${ }^{1} \mathrm{D}$ Walsh ${ }^{*},{ }^{2} \mathrm{G}$ McCartney, ${ }^{3} \mathrm{M}$ Smith, ${ }^{4} \mathrm{G}$ Armour. ${ }^{1} \mathrm{GCPH}$, Glasgow Centre for Population Health, Glasgow, UK; ${ }^{2}$ Public Health Observatory, NHS Health Scotland, Glasgow, UK; ${ }^{3}$ Mental Health Services, NHS Greater Glasgow and Clyde, Glasgow, UK; ${ }^{4}$ Library and Knowledge Services, NHS Health Scotland, Glasgow, UK

10.1136/jech-2019-SSMabstracts.41

Background An increasing amount of evidence has emerged in recent years of the association between different aspects of childhood adversity (termed ACEs - Adverse Childhood Experiences - in a great many studies) and increased risks of negative outcomes in later life. Consequently, ACEs have become a policy priority in many quarters. Despite most ACEs being highly socially patterned, there has been very little discussion in the political discourse regarding the role of childhood socio-economic conditions in understanding and addressing them. The aim, therefore, was to investigate what is known about the association between childhood socio-economic position (SEP) and ACEs, including the extent to which the former explains the latter.

Methods Relevant databases (MEDLINE; psycINFO; ProQuest Public Health Database, Cochrane Library) were searched for all papers satisfying four inclusion criteria: (1) measurement of social position in childhood (prior to ACEs measurement); (2) measurement of multiple aspects of childhood adversity; (3) childhood adversity was the outcome; (4) statistical quantification of the relationship between childhood SEP and childhood adversity. Non-English language papers were excluded.

The initial search terms included ACEs, SEP, and their synonyms. A second search additionally included 'maltreatment'. Papers were independently screened and critically appraised by two authors. Risk of bias was assessed, and overall study quality calculated using a modified version of the Hamilton Tool. Results were synthesised narratively because of the wide variation in definitions of exposures and outcomes.

Results For the ACEs-based search, 2,779 papers were screened, of which 52 were reviewed in full-text. Of these, only six were eligible for qualitative synthesis. The second search (including maltreatment) increased the numbers to: 4,463 papers screened; 166 full texts; 35 included for 